\begin{tabular}{cc}
\hline International Journal of Engineering \&Technology, $7(3.12)(2018) 871-875$ \\
SPC \\
Website: www.sciencepubco.com/index.php/IJET \\
Research paper
\end{tabular}

\title{
Design of High frequency Voltage Controlled Oscillators for Phase Locked Loop
}

\author{
Thejusraj.H ${ }^{1}$, Prithivi Raj ${ }^{2}$, J.Selvakumar ${ }^{3}$, S.Praveen Kumar ${ }^{4}$ \\ Department of Electronics and Communication Engineering, \\ SRM Institute of Science and Technology, SRM Nagar, Potheri, Kattankulathur, \\ Kanchipuram District, Chennai, Tamil Nadu

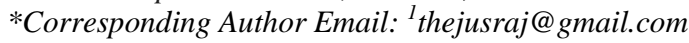

\begin{abstract}
This paper presents the analysis of various oscillators that generate high frequency of oscillation for high speed communication, clock generation and clock recovery. The Ring oscillator and the Current Starved Voltage Controlled Oscillator(CSVCO) (for 5-stagewithout resistor and with resistor) have been implemented using the Cadence Virtuoso tool in $90 \mathrm{~nm}$ technology. The generated frequency of oscillation and the power consumption values of the voltage controlled oscillators have been calculated after inclusion in the PLL, and were also compared to identify the most suitable voltage controlled oscillator for a given application.
\end{abstract}

Keywords: Current Starved VCO, PLL, Ring VCO.

\section{Introduction}

The Phase Locked Loop(PLL) is an integral part of all communication systems. It is a closed loop control system that generates an output signal proportional to the phase of the input signal. It is primarily used for clock synthesis and synchronisation. The Voltage controlled oscillator(VCO) is one of the most vital part of the PLL design as the overall performance characteristics of the PLL is judged based on the performance of the VCO. This paper deals with the performance analysis of the Ring VCO and the Current Starved VCO comprising of both 5 stages and 7 stages.

The section-wise division of this paper is as follows. Section 2 consists of 2 subdivisions. Section 2.1 introduces the concept and circuitry behind the working of Ring VCO. Section 2.2 focuses on a specific type of Ring VCO called the Current Starved Ring VCO of 5 stages. Two variants of the 5 stage VCO, that is one without source degeneration and another with source degeneration are discussed. Section 3 covers the design equations governing the 5 stage Current Starved VCO with source degeneration. Section 4 covers the schematic and simulation results of all the designed VCOs and compares their performance. Section 5 concludes on the preferred design of voltage controlled oscillator for PLL.

\section{Concept and Design}

\subsection{Ring VCO}

The Ring VCO is a feedback system that consists of several delay stages to achieve the required frequency of oscillation. In this paper single ended ring VCO design is implemented.
A single-ended ring VCO comprises of odd number of delay stages. This paper focuses on the 3 stage ring VCO whose circuit is as shown below in Figure1.[3]

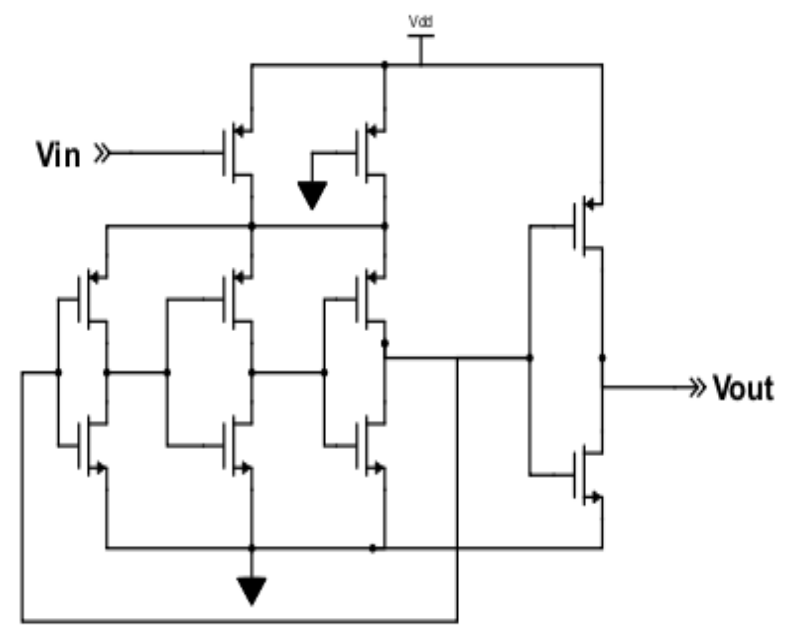

Fig. 1: Ring VCO

Each delay stage consists of two MOSFETs i.e., a PMOS and an NMOS connected to form an inverter. For instance, the MOSFETs M1 and M2 form one delay stage. The gate of M4 is grounded to initiate the oscillation and that of M3 is triggered by a control voltage to control the frequency of output oscillation. In an $\mathrm{N}$ stage ring $\mathrm{VCO}$, each of the delay stages in the ring gives a phase shift of $\pi / N$ and the last inverter stage gives an additional phase shift of $\pi$.

\subsection{Current Starved Ring VCO}

This section explains an improved design of Ring VCO called the Current Starved Ring VCO. In the Current Starved design, unlike 
the basic design of the Ring VCO, there are four MOSFETs in each delay stage as shown in Figure 2.[8]

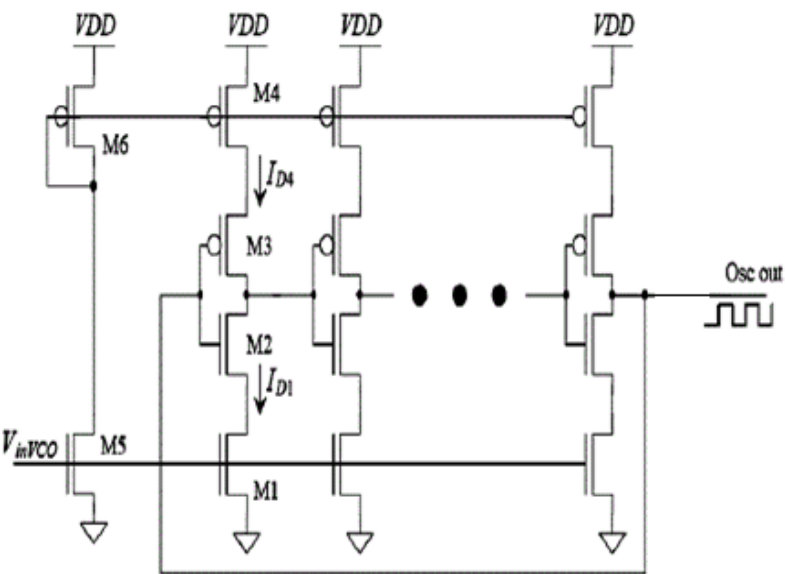

Fig. 2: Current Starved Ring VCO

In Figure2., The PMOSM3 and NMOSM2 form the inverter, while PMOSM4 and NMOSM1 operate as current sources that limit the current available to the inverter i.e., the inverter is starved for current. The current in the NMOS M5 and PMOS M6 are mirrored at each inverter stage.

\subsubsection{Five Stage Current Starved Ring VCO}

The circuit shown in Figure3 is a5 stage Current Starved Ring VCO. There are 5 delay stages.

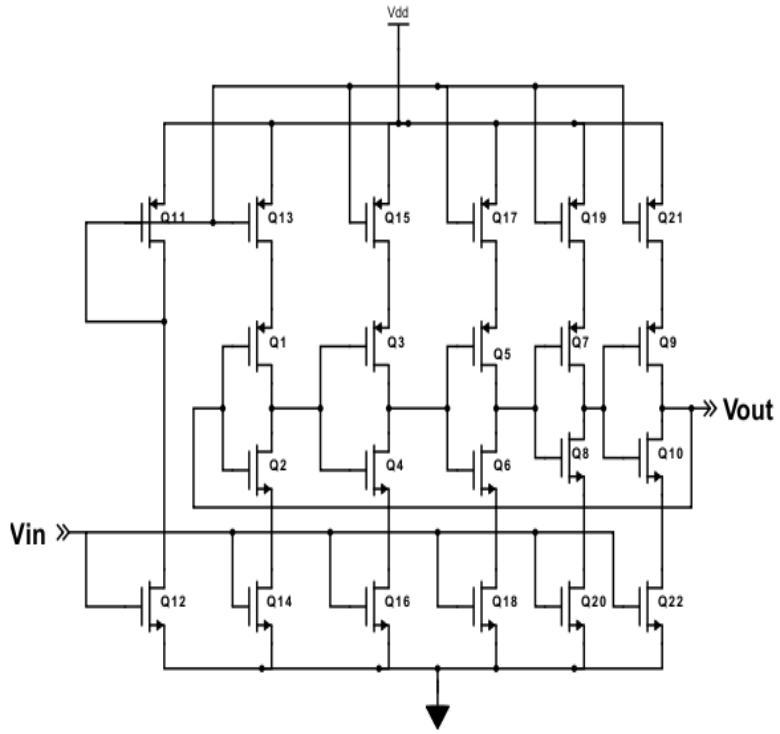

Fig. 3: Five stage CSVCO

In the above circuit, the drain current depends non-linearly on the overdrive voltage. Thus, this circuit is prone to high amount of non-linear distortion with changes in working conditions like temperature.

These drawbacks can be overcome by source degeneration caused by inclusion of a resistor in the design.

\subsubsection{Five Stage Current Starved Ring VCO with Resistor}

The circuit in Figure4 represents the modified 5stage Current Starved Ring VCO after source degeneration.

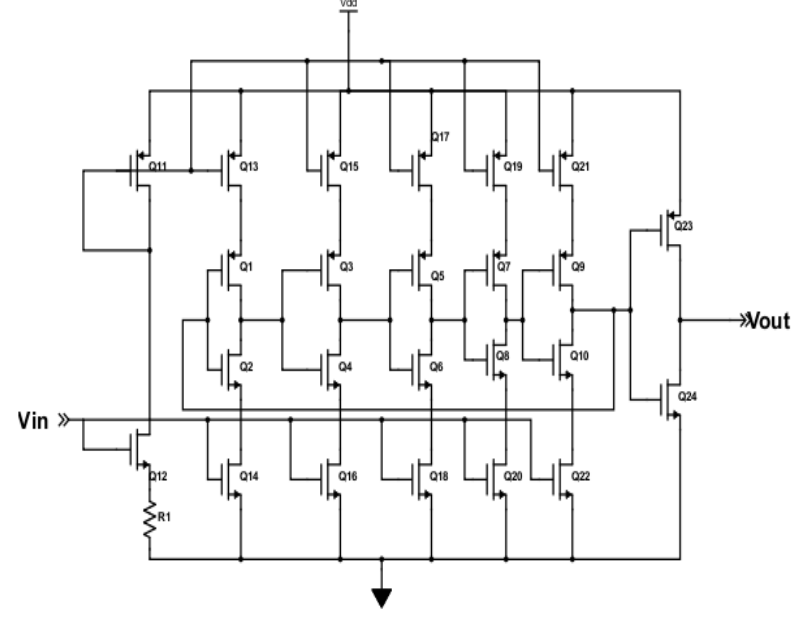

Fig. 4: Five stage CSVCO with resistor

Due to the inclusion of a resistor between the source and ground terminal as shown in Figure 4, the drain current now depends linearly on the overdrive voltage. This circuit now works independent of MOSFET device characteristics and exhibits an overall linear and stable behaviour.

\section{Design Equations}

This section covers the design equations that govern the working of 5 stage CSVCO with resistor.

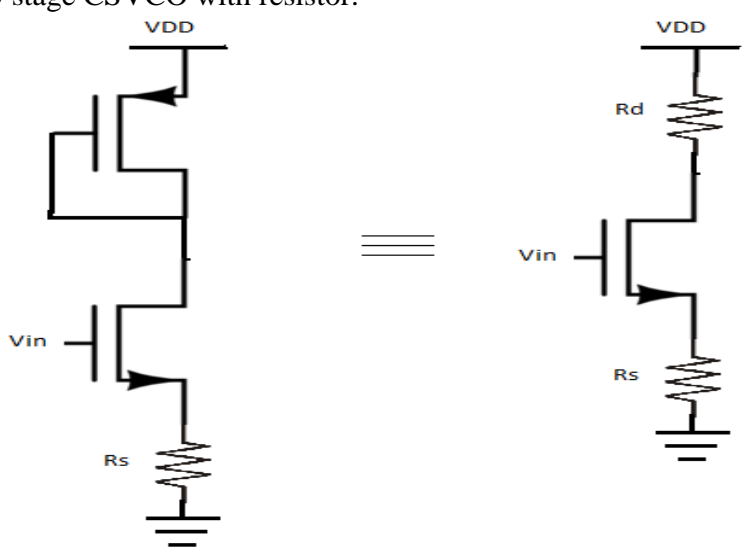

Fig. 5: Source Degeneration

$V_{\text {out }}=-I_{D} R_{d}$

$I_{D}$ (drain current) depends non-linearly on $V_{i n}$ (input voltage). Thus, from equation (1), $V_{\text {out }}$ depends non-linearly on $V_{i n}$, resulting in non-linear behaviour of the circuit.

By source degeneration, transconductance of the circuit is given by equation (2), where $g_{m}$ is the transconductance of the MOSFET.

$G_{m}=\frac{g_{m}}{1+g_{m} R_{s}}$

As $R_{s}$ increases, $G_{m}$ becomes a weakly dependent on $g_{m}$ and hence the drain current $I_{D}$.

For $R_{s} \gg>\left(\frac{1}{g_{m}}\right)$, we get

$G_{m}^{\approx} \frac{1}{R_{S}}$

$\Delta I_{D} \approx \frac{\Delta V_{i n}}{R_{S}}$

Equation (4) indicates that drain current is a linear function of input voltage. 


\section{Simulation Results}

This section covers the schematic of each of the discussed oscillators designed using Cadence Virtuoso tool in $90 \mathrm{~nm}$ technology and their corresponding simulation results.

\subsection{Ring VCO}

Figure 6. is the schematic of the Ring VCO designed using Cadence Virtuoso tool in 90nm technology.

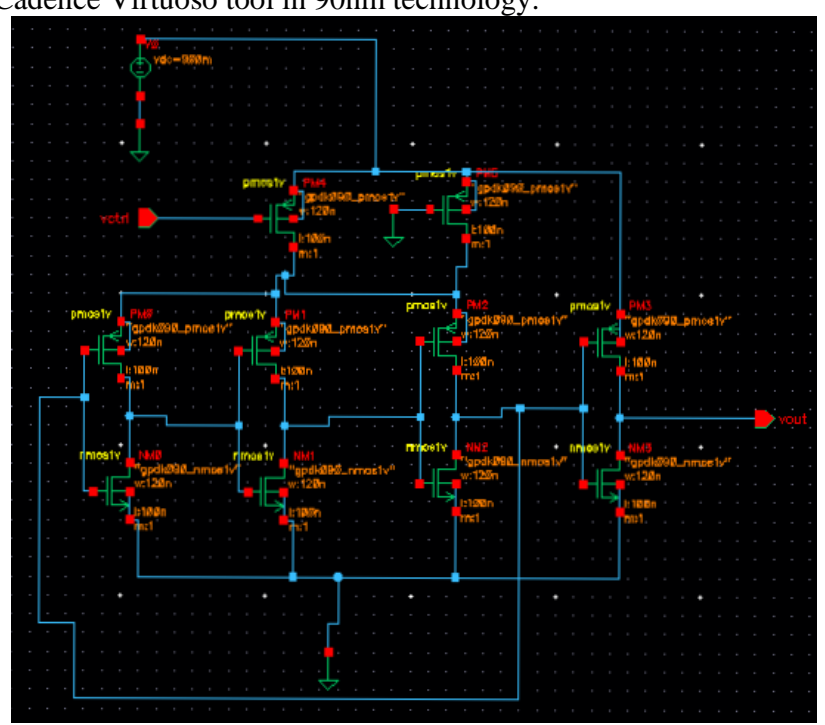

Fig. 6: Ring VCO schematic

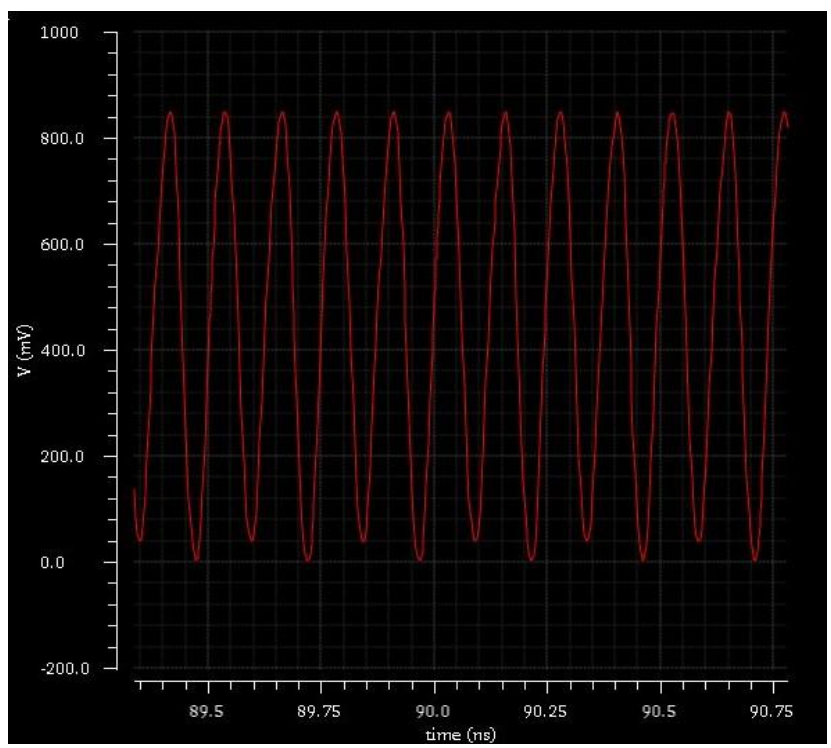

Fig. 7: Ring VCO simulation

Figure 7. is the output of the oscillator. It can be observed that output wave is sinusoidal in nature. The waveform is however not a perfect sine wave. The position of troughs in the wave is fluctuating.

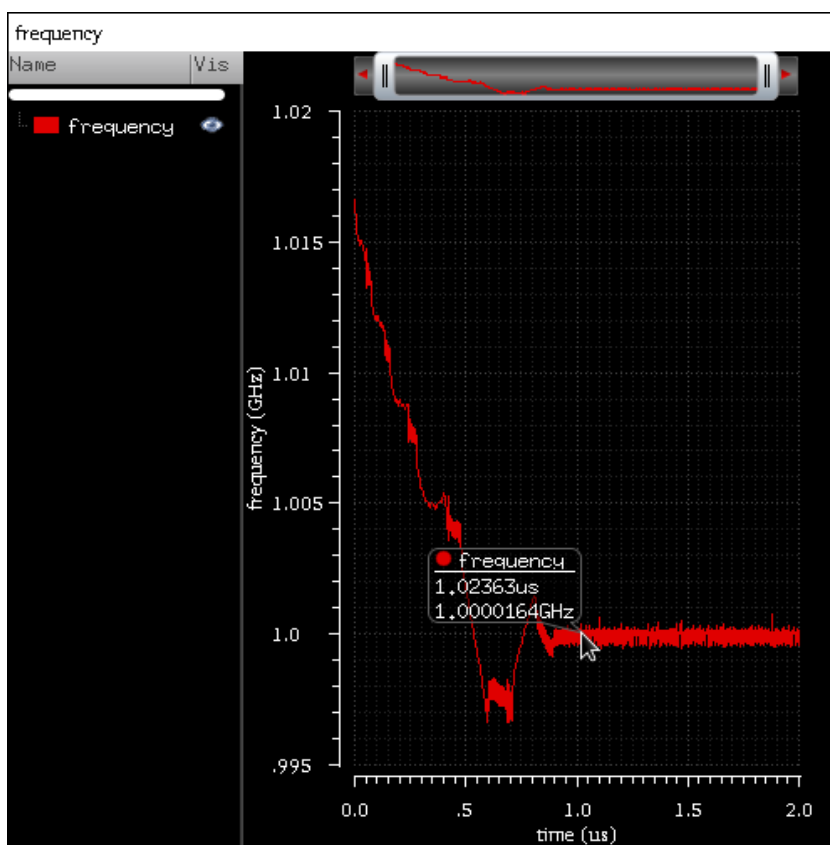

Fig. 8: Ring VCO output frequency plot

The frequency that was achieved is shown in the Figure8., which is around $1 \mathrm{GHz}$. It can be observed that the frequency of oscillation is highly unstable. This was overcome in the latter discussed models of VCO.

\subsection{Five Stage Current Starved VCO without Resistor}

Figure 9. is the schematic of the 5-stage CSVCO without resistor designed using Cadence Virtuoso tool in 90nm technology.

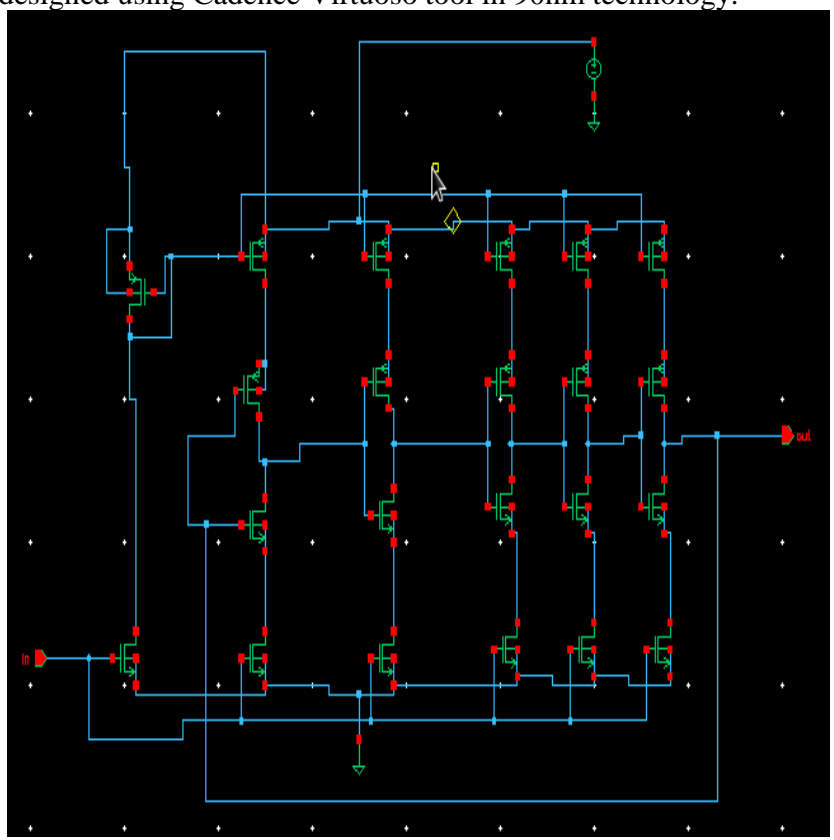

Fig. 9: 5-stage CSVCO (without resistor) schematic 


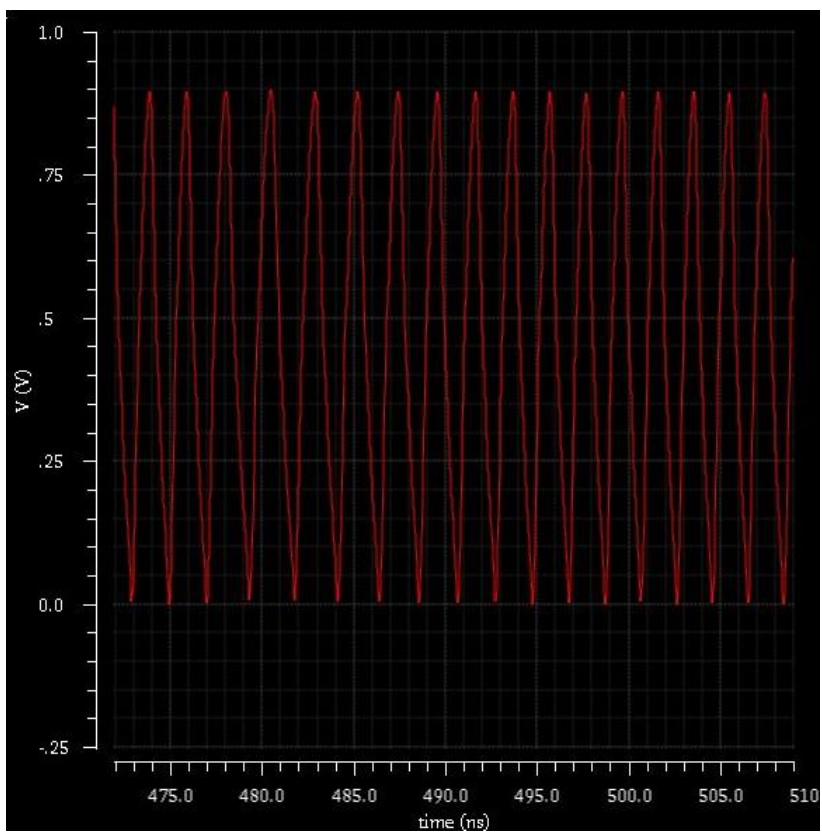

Fig. 10: 5-stage CSVCO (without resistor) simulation

Fig. 10 is the output of the oscillator, after the oscillation frequency has become constant. The output is a sinusoidal wave. The shape of the output waveform is uniform when compared to the output waveform of the previously discussed ring VCO.

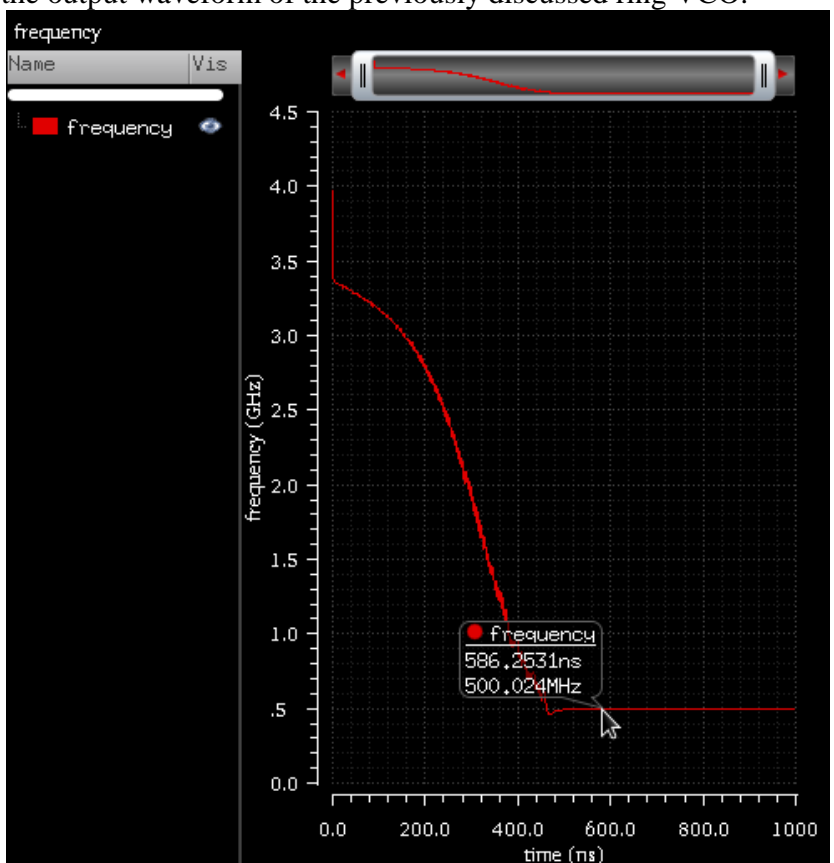

Fig.11: 5-stage CSVCO (without resistor) output frequency plot

The final output frequency attained is shown in the Frequency plot given in Fig.11. The frequency attained is about $500 \mathrm{MHz}$.

\subsection{Five Stage Current Starved VCO with Resistor}

Figure 9. is the schematic of the 5-stage CSVCO with resistor designed using Cadence Virtuoso tool in 90nm technology.

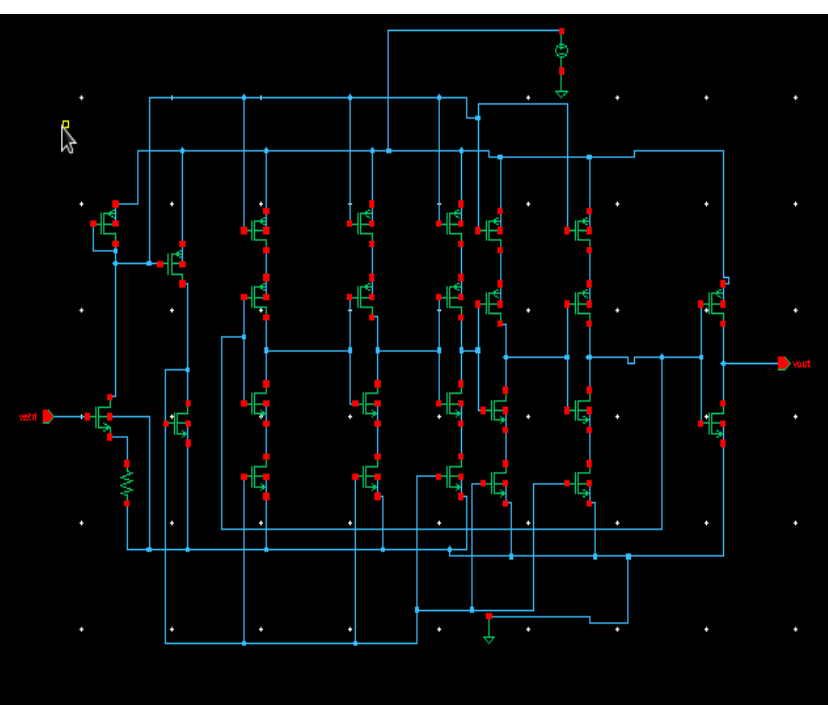

Fig. 12: 5-stage CSVCO (with resistor) schematic

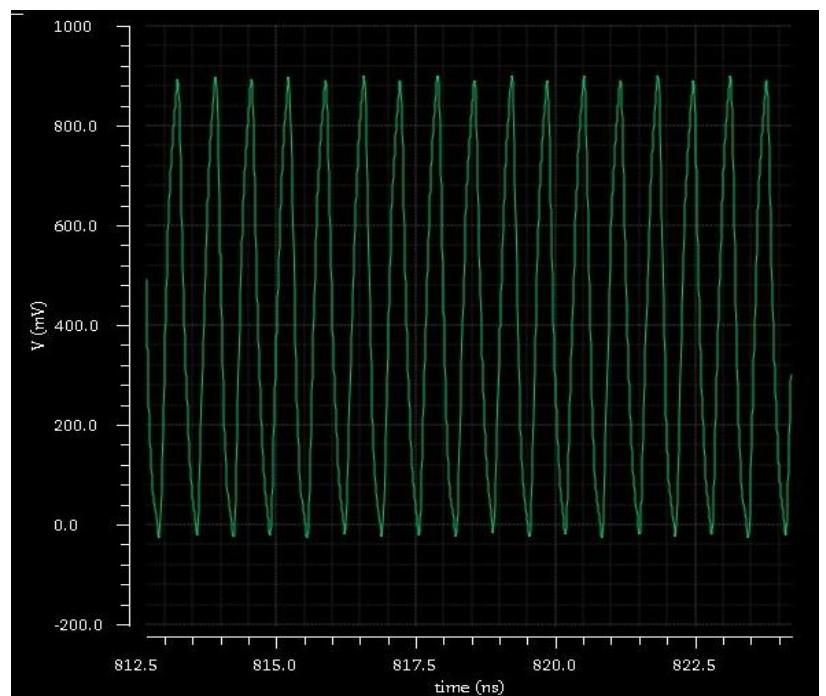

Fig. 13: 5-stage CSVCO (with resistor) simulation

Fig.13 is the output of the oscillator, after the oscillation frequency has become constant. A sinusoidal waveform is obtained.

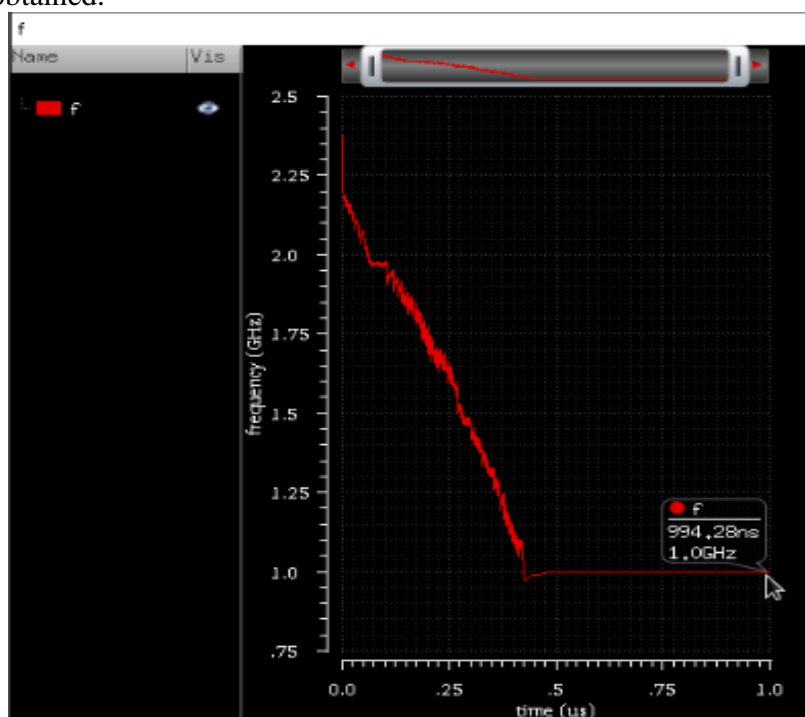

Fig. 14: 5-stage CSVCO (with resistor) output frequency plot

The final output frequency attained is shown in the Frequency plot given in Fig.14.The frequency of output waveform obtained was observed to be $1 \mathrm{GHz}$. 


\subsection{Frequency and Power Consumption Comparison Table}

\begin{tabular}{|c|c|c|}
\hline OSCILLATOR & FREQUENCY & POWER \\
\hline Ring VCO & $1 \mathrm{GHz}$ & $92.27 \mu \mathrm{W}$ \\
\hline $\begin{array}{c}\text { 5 stage CSVCO(without } \\
\text { resistor) }\end{array}$ & $500 \mathrm{MHz}$ & $32.06 \mu \mathrm{W}$ \\
\hline $\begin{array}{c}\text { 5 stage CSVCO(with } \\
\text { resistor) }\end{array}$ & $1 \mathrm{GHz}$ & $72.73 \mu \mathrm{W}$ \\
\hline
\end{tabular}

From the table, it can be observed that the 5 stage CSVCO without resistor consumed the least power i.e., $32.06 \mu \mathrm{W}$ while generating a frequency of $500 \mathrm{MHz}$. On the other hand, the Ring VCO consumed maximum power i.e., $92.27 \mu \mathrm{W}$ while generating a frequency of $1 \mathrm{GHz}$. The 5 stage CSVCO with resistor generated a frequency of $1 \mathrm{GHz}$ with a power consumption of $72.73 \mu \mathrm{W}$ that is intermediate to the other VCOs.

\section{Conclusion}

The comparison in the performance of the Ring VCO, 5 stage Current Starved Ring VCO with resistor and without resistor has been done in terms of frequency and power consumption using Cadence Virtuoso tool in $90 \mathrm{~nm}$ technology. Each of these oscillators where placed in the PLL to conclude on the best fit for the application of high speed communication. It was observed that the Current Starved design of Ring VCO was better than the basic design of the Ring VCO in terms of power consumption. In order to achieve higher frequencies, the number of stages in the CSVCO can be increased. With the inclusion of resistance in the 5 stage CSVCO, a linear and stable behaviour of the circuit is observed. Also the frequency of oscillation is higher, however with a tradeoff in power consumption.

\section{References}

[1] Saikee Chauhan, , Rajesh Mehra, " CMOS Design and Performance Analysis of Ring Oscillator for Different Stages", International Journal of Engineering Trends and Technology (IJETT) - Volume 32 Number 5- February 2016.

[2] Yashpal Sen, Nitin Jain, " Design and Implementation of Phase Locked Loop Using Current Starved Voltage Controlled Oscillator", Advance in Electronic and Electric Engineering. ISSN 2231-1297, Volume 4, Number 6 (2014).

[3] Rishi Ratan" Design of A Phase Locked Loop Based Clocking Circuit For High Speed Serial Link Applications" University of Illinois at Urbana-Champaign, 2014

[4] B. Razavi, "Design of Analog CMOS Integrated Circuits," Tata McGraw Hill Edition, 2002.

[5] Shruti Suman, K.G Sharma, P.K Ghosh, "Analysis and Design of Current Starved Ring Vco", International Conference on Electrical, Electronics and Optimisation Techniques (ICEEOT)-2016

[6] M.D Sudara, V.S Wijesinghe, D.M Serasinghe, J.G.D.A Thilakaratne and Subramaniam Thayaparan "Implementation and Analysis of Fast Locking 5GHz Phase Locked Loop" 2016 IEEE

[7] G.Jovanovi'c,M.Stoj cev,Z.Stamenkovic, "A CMOS Voltage Controlled Ring Oscillator with Improved Frequency Stability" Scientific Publications of The State University of Novi Pazar SER. A: APPL. MATH. INFORM. AND MECH. vol. 2, 1 (2010), 1-9.

[8] MalothuDilipKummarNaik, Design and Analysis of Efficient Phase Locked Loop, Department of electronics and Communication, National Institute of Technology, Rourkela 2015.

[9] Shobha K M, Sudhakara H M, Praveen J, Raghavendra Rao A, " Design and Analysis of Phase Locked Loop based Frequency Synthesizer using Source Coupled VCO", International Journal of Innovative Research in Electrical, Electronics, Instrumentation and Control engineering", Vol. 4, Issue 5, May 2016.

[10] Sidhartthsaxena, M.Srikanth, et.al, "Efficient VCO using Finfet", Indian Journal of Science and Technology", Vol8(S2), 262-270, JAnuary 2015.

[11] Madhusudan Kulkarni and Kalmeshwar N Hosur, "Design of a linear and wide range Current starved Voltage Controlled Oscillator for PLL", International Journal on Cybernetics \& Informatics ( IJCI) Vol.2, No.1, February 2013.
[12] R. Jacob Baker. CMOS circuit design, Layout and simulation, John Wiley and Sons Inc, Publication, 2010.

[13] Roland E. Best, Phase-Locked Loops Design, Simulation and Applications, 5th edition, McGraw- Hill Publications.

[14] Stanley Goldman, Phase-Locked Loop Engineering Handbook for Integrated Circuits, Artech House, Inc, 2007.

[15] Cadence manual, 2013. 